\title{
Vibration Transmission of a Cylindrical Shell with an Interior Rectangular Plate with the Receptance Method
}

\author{
Zhimei Zhao, Meiping Sheng, and Yang Yang \\ School of Marine Engineering, Northwestern Polytechnical University, Shaanxi, Xi'an 710072, China \\ Correspondence should be addressed to Zhimei Zhao, zhimeizhao@gmail.com
}

Received 3 May 2012; Accepted 7 August 2012

Academic Editor: Rama Bhat

Copyright () 2012 Zhimei Zhao et al. This is an open access article distributed under the Creative Commons Attribution License, which permits unrestricted use, distribution, and reproduction in any medium, provided the original work is properly cited.

\begin{abstract}
Vibration transmission characteristics of a cylindrical shell with a rectangular plate are discussed in this paper by the substructure receptance method. The system is divided into two substructures: the plate and the shell. After finding the theoretical receptance function of each substructure, the coupling equation of the combined system is solved by considering the continuity conditions at the joint between the plate and the shell. The numerical results are compared with the experimental ones to show the validity of the formulation. After that, effects of the plate's parameters on vibration transmission characteristics are discussed. The parameter study shows that it is important to avoid impedance matching for the plate and the shell in the design of suitable structures with low vibration and noise radiation characteristics.
\end{abstract}

\section{Introduction}

Plates and shells are widely used as basic components in many engineering structures, such as aircrafts [1] and underwater structures [2]. Until now, their fundamental physical mechanisms and phenomenons, such as free vibration [3], wave propagation $[4,5]$, and power flow [6], have been discussed intensely. Plates and shells are often utilized by coupling together with welds, bolts, or dampers in practical applications. Dynamic behaviors of these coupled structures become relatively complicated due to vibration energy transmission between the interior plate and the shell. Less literature is available on these topics. However, as vibration transferred from the plate to the shell would result in the shell's outward noise radiation; it is of great importance for engineers to understand these combined structures' vibration, especially the effects of parameters on the combinations' dynamic behaviors, which is helpful for designing suitable structures with low vibration and noise radiation. Forced vibration of one variant of these combined structures, a partitioned cylindrical shell with a longitudinal, interior rectangular plate, has been investigated in this paper to simulate an aircraft fuselage with an interior floor.

Vibroacoustic characteristics of a cylindrical shell with an interior rectangular plate have been studied in several papers, which mainly focused on free vibration analysis. The first analytical model was developed by Peterson and Boyd [7] with applications of Rayleigh-Ritz technique. Effects of structural parameters, including rigid joint, hinged joint, thickness and position of the floor on natural frequencies and mode shapes were analyzed. Irie et al. [8] studied free vibration of noncircular cylindrical shells with longitudinal interior partitions by using the transfer matrix. Langley [9] studied free vibration of a simply supported stiffened cylindrical shell with an internal plate by a dynamic stiffness method, where three structural elements were used. Missaoui et al. [10] investigated free and forced vibration of a cylindrical shell with a floor partition based on a variation formulation in which the structural coupling was simulated with an artificial spring system. Missaoui and Cheng [11] and $\mathrm{Li}$ et al. [12] presented findings on the structural acoustic coupling characteristics of a cylindrical shell with an internal floor partition. Missaoui also pointed out that the receptance method might be difficult to exploit for a shell partitioned by a longitudinal floor since no information on forces distributing along the joint is priori available. However, Lee et al. [13, 14] assumed the line forces and moments applied along the joint as sinusoidal functions and discussed the free vibration of an isotropic and homogeneous shell with an interior rectangular plate, and a laminated 
composite cylindrical shell with a plate using the receptance method. He proved that the receptance method is efficient for free vibration of a shell/plate-combined system. Yet only the transverse force and moment were considered, which might be inaccurate. Wang et al. [15] studied power flows in a coupled plate-cylindrical shell system by the substructure method with consideration of in-plane forces, transverse forces, and moments, where receptance functions of the plate and the shell were also needed.

Descriptions above demonstrate that one of the most efficient approaches for analyzing the vibration of the combined structure is the receptance method, which has been reported extensively in the literature. Vibration energy transmission takes place even in simple structures between different parts of the structure. The complexity is in our inability to define the behavior of the structure with a single equation as in the case of a shell or a plate or a beam, and so forth. Moreover, the receptance method has been in use since as early as 1960 when Bishop and Johnson [16] describe the method. It was used by Azimi et al. [17] to find mode shapes and natural frequencies for free vibration of coupled rectangular plates. Huang and Soedel [18-20] utilized this method to analyze both free and forced vibration of a simply supported cylindrical shell coupled with annular plates. Tao et al. [21] analyzed the force transmission from a rigid body mounting system to a flexible foundation and used the receptance matrix to derive the analytical expression. Tursun and Eskinat [22] found that the receptance method is efficient for the minimization of the unwanted vibration in engineering. This method obtains interface coupling forces by considering geometrical compatibility conditions and force balance equations at the shell/plate joint. Global vibration information of the combined structure can be synthesized in terms of each substructure's receptance functions, which can be calculated by the modal expansion method [23], separately. Thus, the receptance method is simple and efficient and gives more physical insight than the purely numerical ones. However, it has not been applied for forced vibration analysis of a cylindrical shell with an interior plate until now. This paper further developed the receptance method to examine the forced vibration characteristics of a simply supported cylindrical shell with an interior rectangular plate.

Previous studies $[7,13,14]$ have demonstrated that the fundamental frequency of the combined plate/shell system exhibits principally plate motion with one half wave in each direction. The plate modes of the coupled system in the lower range increase as the plate is located farther from the center of the shell due to the increment of the plate stiffness. Thus, the plate's stiffness has great influence on free vibration of the cylindrical shell with an interior rectangular plate. Though some investigation on such plate/shellcoupled system has been carried out, little attention seems to have been focused on the plate's influence on vibration transmission between the floor and the shell, which is of great importance in designing quiet aircraft as it plays a key point in understanding the plate/shell coupled system's vibration behavior. Thus it is discussed here. First, the theory model of a cylindrical shell coupled conservatively with a rectangular plate is established by the receptance method. Then results obtained by the receptance method are compared with those of FEM model to validate the model. Last, numerical examples are provided to illustrate the effect of the plate's parameters on vibration transmission characteristics of the system.

\section{Analytical Approach}

The partitioned cylindrical shell with an interior plate is illustrated in Figure 1. The plate is attached at $\theta_{1}^{*}$ and $\theta_{2}^{*}$ position of the shell based on the horizontal centerline. It is assumed that shear diaphragm-shear diaphragm (SD-SD) boundary conditions apply to the ends of the plate and the shell. Both the plate and the cylindrical shell are assumed to be free at their coupling edges. The coupled structure can be divided into two substructures: the plate and the shell. They are coupled together by the internal forces $\left[\mathbf{F}_{c s i}\right]=$ $\left[q_{i}^{s N}, q_{i}^{s Q}, q_{i}^{s M}\right]^{T}(i=1,2)$, as shown in Figure 2, distributing along the coupling edges of the shell and internal forces $\left[\mathbf{F}_{c p i}\right]=\left[q_{i}^{p N}, q_{i}^{p Q}, q_{i}^{p M}\right]^{T}(i=1,2)$, as shown in Figure 3, distributing along the coupling edges of the plate. Besides, the plate is also excited by external forces $\left[\mathbf{F}_{p e}\right]$ while the shell is excited by external forces $\left[\mathbf{F}_{s e}\right]$.

2.1. Coupling Equations. A receptance is defined as the ratio of a steady-state displacement or slope response at a certain point to a harmonic line force or line moment input at the same (direct receptance) or at a different point (crossreceptance) [20]. It is employed here to formulate vibration equations of each substructure.

As described above, the shell is excited by the internal distributed forces $\left[\mathbf{F}_{s c i}\right](i=1,2)$ and the external point force $\left[\mathbf{F}_{s e}\right]$. According to the receptance method, the shell's motion equation can be written as

$$
\left[\mathbf{u}_{s}\right]=\left[\mathbf{Y}_{s c 1}\right]\left[\mathbf{F}_{s c 1}\right]+\left[\mathbf{Y}_{s c 2}\right]\left[\mathbf{F}_{s c 2}\right]+\left[\mathbf{Y}_{s e}\right]\left[\mathbf{F}_{s e}\right]
$$

where $\left[\mathbf{u}_{s}\right]=\left[v_{s}, w_{s}, \psi_{s}\right]^{T}$ is the shell's dynamic displacement vector, and its direction is corresponding to that of the internal forces $\left[\mathbf{F}_{s c i}\right]$ in the shell's local coordinate; $\left[\mathbf{Y}_{s c i}\right](i=$ $1,2)$ are the receptance matrices corresponding to $\left[\mathbf{F}_{s c i}\right](i=$ $1,2) ;\left[\mathbf{Y}_{s e}\right]$ is the receptance matrices corresponding to the external force $\left[\mathbf{F}_{s e}\right]$.

The plate is excited by the internal distributed forces $\left[\mathbf{F}_{p c i}\right](i=1,2)$ and the external point force $\left[\mathbf{F}_{p e}\right]$. Analogously, its motion equation can be written as

$$
\left[\mathbf{u}_{p}\right]=\left[\mathbf{Y}_{p c 1}\right]\left[\mathbf{F}_{p c 1}\right]+\left[\mathbf{Y}_{p c 2}\right]\left[\mathbf{F}_{p c 2}\right]+\left[\mathbf{Y}_{p e}\right]\left[\mathbf{F}_{p e}\right],
$$

where $\left[\mathbf{u}_{p}\right]=\left[v_{p}, w_{p}, \psi_{p}\right]^{T}$ is the dynamic displacement vector of the plate, and its direction is the same with that of the internal forces $\left[\mathbf{F}_{p c i}\right] ;\left[\mathbf{Y}_{p c i}\right](i=1,2)$ and $\left[\mathbf{Y}_{p e}\right]$ are the related receptance matrices of the plate.

When a cylindrical shell is coupled with an interior rectangular plate rigidly, no energy would dissipate at their joint. Thus, their coupling is conservative. Then the geometrical compatibility conditions and force balance equations at the 


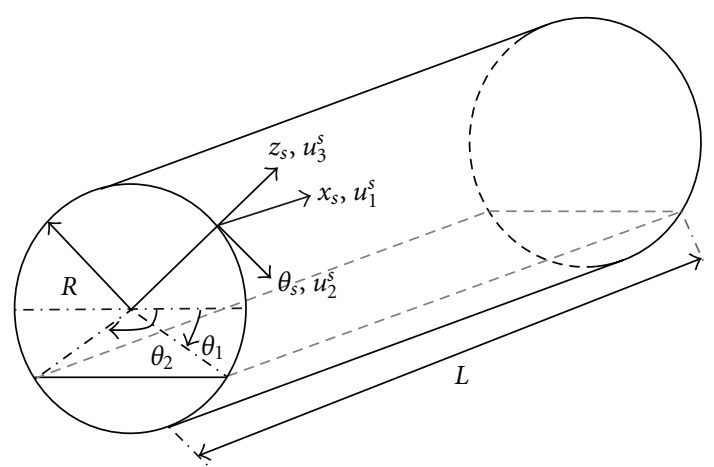

FIGURE 1: Shell-plate coupled structure.

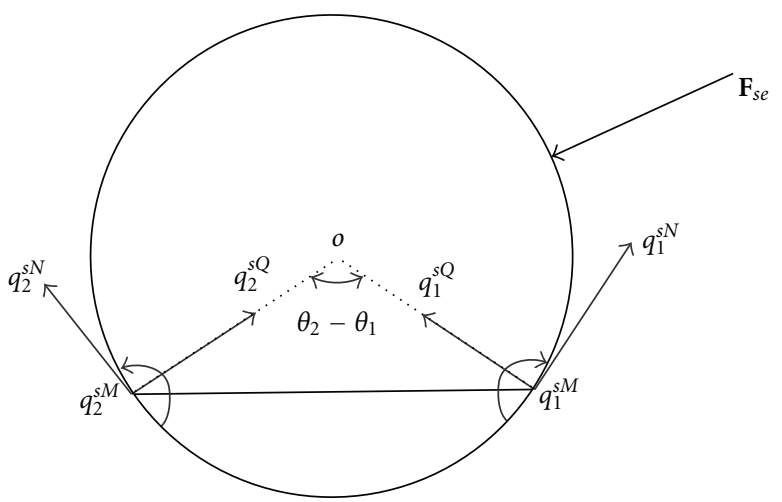

FIgURE 2: Forces applied on the shell.

shell/plate joints, in the global coordinate system, can be demonstrates by equations as follows:

$$
\begin{gathered}
{\left.\left[\mathbf{u}_{p}\right]\right|_{l_{i}}-\left.\left[\mathbf{T}_{i}\right]\left[\mathbf{u}_{s}\right]\right|_{l_{i}}=0 \quad(i=1,2),} \\
{\left[\mathbf{F}_{p c i}\right]+\left[\mathbf{T}_{i}\right]\left[\mathbf{F}_{s c i}\right]=0 \quad(i=1,2),}
\end{gathered}
$$

where $\left[\mathbf{T}_{i}\right](i=1,2)$ represents the transformation matrices from the shell's local cylindrical coordinate system to the plate's local Cartesian coordinates system, defined at the two coupling edges respectively; subscript $l_{i}$ denotes coupling edge $i$.

Substituting (1), (2), and (3) into (4), coupling forces of the shell would be expressed as

$$
\begin{aligned}
{\left[\begin{array}{ll}
\mathbf{F}_{s c 1} & \mathbf{F}_{s c 2}
\end{array}\right]^{T}=} & -\left([\mathbf{T}]\left[\mathbf{Y}_{s c}\right]+\left[\mathbf{Y}_{p c}\right][\mathbf{T}]\right)^{-1} \\
& \times\left([\mathbf{T}]\left[\mathbf{Y}_{s e}\right]\left[\mathbf{F}_{s e}\right]-\left[\mathbf{Y}_{p e}\right]\left[\mathbf{F}_{p e}\right]\right),
\end{aligned}
$$

where

$$
\begin{gathered}
{[\mathbf{T}]=\left[\begin{array}{lll}
\mathbf{T}_{1} & \mathbf{0} ; \mathbf{0} & \mathbf{T}_{2}
\end{array}\right]} \\
{\left[\mathbf{Y}_{s c}\right]=\left[\begin{array}{lll}
\left.\mathbf{Y}_{s c 1}\right|_{l_{1}} & \left.\mathbf{Y}_{s c 2}\right|_{l_{1}} ;\left.\mathbf{Y}_{s c 1}\right|_{l_{2}} & \left.\mathbf{Y}_{s c 2}\right|_{l_{2}}
\end{array}\right],} \\
{\left[\mathbf{Y}_{p c}\right]=\left[\begin{array}{lll}
\left.\mathbf{Y}_{p c 1}\right|_{l_{1}} & \left.\mathbf{Y}_{p c 2}\right|_{l_{1}} ;\left.\mathbf{Y}_{p c 1}\right|_{l_{2}} & \left.\mathbf{Y}_{p c 2}\right|_{l_{2}}
\end{array}\right]}
\end{gathered}
$$

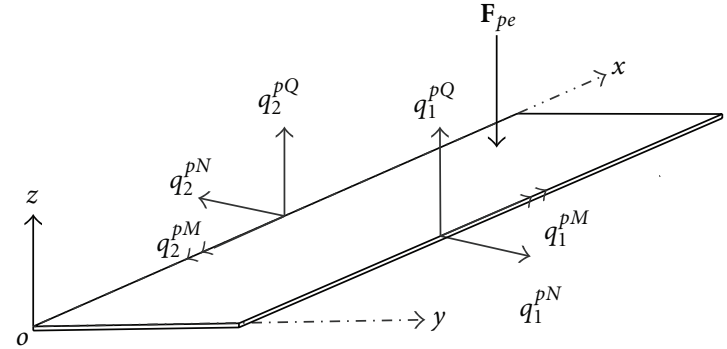

Figure 3: Forces applied on the plate.

$$
\begin{gathered}
{\left[\mathbf{Y}_{s e}\right]=\left[\begin{array}{ll}
\left.\mathbf{Y}_{s e}\right|_{l_{1}} & \left.\mathbf{Y}_{s e}\right|_{l_{2}}
\end{array}\right]^{T},} \\
{\left[\mathbf{Y}_{p e}\right]=\left[\begin{array}{ll}
\left.\mathbf{Y}_{p e}\right|_{l_{1}} & \left.\mathbf{Y}_{p e}\right|_{l_{2}}
\end{array}\right]^{T} .}
\end{gathered}
$$

It is possible to get the coupling forces $\left[\mathbf{F}_{c s i}\right](i=1,2)$ through (5) once receptance functions of the plate and the shell have been derived, respectively. Then, internal forces $\left[\mathbf{F}_{c p i}\right](i=1,2)$, which are applied to the plate, can be obtained through (4). After that, response of the shell/plate coupled system can be determined by taking $\left[\mathbf{F}_{c s i}\right](i=1,2)$ and $\left[\mathbf{F}_{c p i}\right](i=1,2)$ into $(1)$ and $(2)$.

In engineering, excitations for such coupled structures are often applied at the plate. For the application here, only an external harmonic external force $\left[\mathbf{F}_{p e}\right]$ is assumed to act on the plate. Thus, the shell is only excited by the internal forces $\left[\mathbf{F}_{c s i}\right](i=1,2)$. Therefore, the time-averaged input power $P_{\text {in }}$ from this external force to the combined structure can be obtained easily. According to the basic definition of the power flow in a continuum [23], the timeaveraged transmitted power $P_{\text {trans }}$ at the coupling edges can be acquired and expressed as

$$
P_{\text {trans }}=\frac{1}{2} \int_{0}^{L} \operatorname{Re}\left\{\left[\begin{array}{ll}
\mathbf{F}_{c s 1} & \mathbf{F}_{c s 2}
\end{array}\right]\left[\begin{array}{ll}
\left.\dot{\mathbf{u}}_{c s}^{*}\right|_{l_{1}} & \left.\dot{\mathbf{u}}_{c s}^{*}\right|_{l_{2}}
\end{array}\right]^{T}\right\} \mathrm{d} x,
$$

where $\dot{\mathbf{u}}_{c s i}^{*}(i=1,2)$ denotes differentiation with respect to time and then takes the complex conjugate value; $L$ is the length of the cylindrical shell.

According to the theory of statistical energy analysis (SEA), the spatial averaged surface velocity of the shell can be determined by approximately equaling the rate of the energy dissipation of the shell due to the internal loss factor. Thus the shell's spatial averaged acceleration $\overline{a_{s}}$ can be expressed as

$$
\left|\overline{a_{s}}\right| \approx \sqrt{\frac{\omega P_{\text {trans }}}{\eta_{s} M_{s}}},
$$

where $\omega$ is the annular frequency; $\eta_{s}$ and $M_{s}$ are the shell's loss factor and mass.

As the source plate is only connected to the shell, the energy dissipation of the plate equals the subtraction of the input power $P_{\text {in }}$ and the transmitted power $P_{\text {trans }}$. Therefore, the plate's spatial averaged acceleration $\overline{a_{p}}$ can be expressed as

$$
\left|\overline{a_{p}}\right| \approx \sqrt{\frac{\omega\left(P_{\text {in }}-P_{\text {trans }}\right)}{\eta_{p} M_{p}}},
$$

where $\eta_{p}$ and $M_{p}$ are the loss factor and the mass of the plate. 


\subsection{Receptances of the Shell and the Plate}

2.2.1. Steady Harmonic Response. To solve (5), it is essential to get the plate's and the shell's related receptance matrices $\left[\mathbf{Y}_{s c i}\right],\left[\mathbf{Y}_{s e}\right],\left[\mathbf{Y}_{p c i}\right]$, and $\left[\mathbf{Y}_{p e}\right]$. Thus, it is prior to get substructure's steady-state harmonic response. As shown in Figures 2 and 3, each substructure is treated as an idealized system consisting of a single rectangular uniform plate or an isotropic cylindrical shell. Their structural damping properties are represented by a linear Voigt viscoelastic model [24] with loss factor $\eta$, which is assumed to be the same in all three principal directions to offer computational advantages. Forces applied on substructures are assumed to be independent of the motion of the shell, which is an admissible approximation for most engineering cases.

For continuous substructures, the number of degrees of freedom is infinite. Thus, displacements of the shell or the plate subjected to dynamic loading can be expressed by modal displacement and modal participation factor as an infinite series as follows:

$$
u_{i}\left(\alpha_{1}, \alpha_{2}, t\right)=\sum_{m n} \lambda_{m n}(t) U_{i m n}\left(\alpha_{1}, \alpha_{2}\right), \quad i=1,2,3,
$$

where $\alpha_{1}, \alpha_{2}$ is the two-dimensional curvilinear surface coordinates, for the shell $\alpha_{1}=x, \alpha_{2}=\theta$ and for the plate $\alpha_{1}=x, \alpha_{2}=y ; U_{\text {imn }}(i=1,2,3)$ denote the mode components of the substructure in the three principal directions; $m, n$ represents the mode number. The modal participation factor $\lambda_{m n}(t)$ is the root of the following modal equation for steady-state harmonic response of the structure:

$$
\ddot{\lambda}_{m n}+2 \xi_{m n} \omega_{m n} \dot{\lambda}_{m n}+\omega_{m n}^{2} \lambda_{m n}=F_{m n}^{*} e^{j \omega t}
$$

where

$$
\begin{aligned}
N_{m n}=\int_{\alpha_{2}} \int_{\alpha_{1}}( & U_{1 m n}^{2}\left(\alpha_{1}, \alpha_{2}\right)+U_{3 m n}^{2}\left(\alpha_{1}, \alpha_{2}\right) \\
& \left.+U_{3 m n}^{2}\left(\alpha_{1}, \alpha_{2}\right)\right) A_{1} A_{2} d \alpha_{1} d \alpha_{2}
\end{aligned}
$$

If $q_{i}$ is force,

$$
F_{m n}^{*}=\frac{1}{\rho h N_{m n}} \int_{\alpha_{2}} \int_{\alpha_{1}} q_{i}^{*}\left(\alpha_{1}, \alpha_{2}, t\right) U_{i m n}\left(\alpha_{1}, \alpha_{2}\right) A_{1} A_{2} d \alpha_{1} d \alpha_{2}
$$

If $q_{i}$ is the distributed moment around the $\alpha_{1}$-axis direction,

$$
F_{m n}^{*}=\frac{1}{\rho h N_{m n}} \int_{\alpha_{2}} \int_{\alpha_{1}} \frac{\partial q_{i}^{*}\left(\alpha_{1}, \alpha_{2}, t\right)}{\partial \alpha_{2}} U_{3 m n}\left(\alpha_{1}, \alpha_{2}\right) A_{1} d \alpha_{1} d \alpha_{2} .
$$

In (16), $\xi_{m n}=\eta /\left(\rho h \omega_{m n}\right)$ is the equivalent viscous damping factor, $\eta$ is the loss factor, $\rho$ and $h$ denote mass density and thickness of the related substructure; $\omega_{m n}$ is natural frequency; $A_{1}$ and $A_{2}$ are the fundamental form parameters and $A_{1}=A_{2}=1$ for the plate, $A_{1}=1, A_{2}=R$ for the shell; $R$ is the radius of the shell; $j=\sqrt{-1}$. The input forcing functions $q_{i}^{*}(i=1,2,3)$ are the forces applied in the three principal directions.
At steady state, the dynamic response will also be harmonic. Thus, the mode participation factor in (16) can be obtained as

$$
\lambda_{m n}(t)=\frac{F_{m n}^{*} e^{j \omega t}}{\rho h N_{m n}\left(\omega_{m n}^{2}-\omega^{2}+2 j \xi_{m n} \omega_{m n} \omega\right)} .
$$

2.2.2. Shell Receptance. The cylindrical shell discussed here is supposed to be thin and isotropic. According to Love shell theory [22], under the influence of harmonic excitation and modal analysis, the displacement functions which satisfy the SD-SD boundary conditions at both ends are of the following form:

$$
\begin{aligned}
u_{1}^{s}(x, \theta, t) & =\sum_{i=1}^{3} \sum_{m=0}^{\infty} \sum_{n=1}^{\infty} \lambda_{m n i}^{s}(t) U_{1 m n i}^{s}(x, \theta) \\
& =\sum_{i=1}^{3} \sum_{m=0}^{\infty} \sum_{n=1}^{\infty} \lambda_{m n i}^{s}(t) \frac{A_{m n i}}{C_{m n i}} \cos m(\theta-\phi) \cos \left(k_{n} x\right), \\
u_{2}^{s}(x, \theta, t) & =\sum_{i=1}^{3} \sum_{m=0}^{\infty} \sum_{n=1}^{\infty} \lambda_{m n i}^{s}(t) U_{2 m n i}^{s}(x, \theta) \\
& =\sum_{i=1}^{3} \sum_{m=0}^{\infty} \sum_{n=1}^{\infty} \lambda_{m n i}^{s}(t) \frac{B_{m n i}}{C_{m n i}} \sin m(\theta-\phi) \sin \left(k_{n} x\right), \\
u_{3}^{s}(x, \theta, t) & =\sum_{i=1}^{3} \sum_{m=0}^{\infty} \sum_{n=1}^{\infty} \lambda_{m n i}^{s}(t) U_{3 m n i}^{s}(x, \theta) \\
& =\sum_{i=1}^{3} \sum_{m=0}^{\infty} \sum_{n=1}^{\infty} \lambda_{m n i}^{s}(t) \cos m(\theta-\phi) \sin \left(k_{n} x\right),
\end{aligned}
$$

where $U_{1 m n}^{s}, U_{2 m n}^{s}$ and $U_{3 m n}^{s}$, are the cylindrical shell's natural vibration modes in $x, \theta, z$ directions, respectively; $k_{n}=n \pi / L$ and $L$ is the length of the cylindrical shell; $m$ and $n$ represent half wave numbers in the $\theta$ and $x$ directions, respectively; $\phi$ is the arbitrary angle. Expressions of $A_{i} / C_{i}$ and $B_{i} / C_{i}$ can be found in [22].

Take (20) into (16), then

$$
\begin{aligned}
& N_{m n i}^{s} \\
& = \begin{cases}{\left[\left(\frac{A_{m n i}}{C_{m n i}}\right)^{2}+\left(\frac{B_{m n i}}{C_{m n i}}\right)^{2}+1\right] \frac{\pi R L}{2},} & m \neq 0, n \neq 0, \\
{\left[\left(\frac{A_{m n i}}{C_{m n i}}\right)^{2}+1\right] \pi R L,} & m=0, n \neq 0 .\end{cases}
\end{aligned}
$$

For the plate-shell-coupled system analyzed here, the rectangular plate is joined to the shell at $\theta=\theta_{1}$ and $\theta=$ $\theta_{2}$. The line loads applied to the shell corresponding to each plate-shell joint are the forces $q_{k}^{s N}(x, \theta, t)(k=1,2)$ in circular direction, $q_{k}^{s F}(x, \theta, t)(k=1,2)$ in radial direction and moments and $q_{k}^{s M}(x, \theta, t)(k=1,2)$ around the $x$-axis direction. Assume that all the internal forces and moments distribute sinusoidally along the coupling edges. Then the 
line loads exerted on the shell at the joint, as shown in Figure 2, can be expressed as follows:

$$
\begin{array}{ll}
q_{k}^{s N}(x, \theta, t)=N_{k}^{s} \sin \left(k_{n^{\prime}} x\right) \delta\left(\theta-\theta_{k}\right) e^{j \omega t} & (k=1,2), \\
q_{k}^{s Q}(x, \theta, t)=Q_{k}^{s} \sin \left(k_{n^{\prime}} x\right) \delta\left(\theta-\theta_{k}\right) e^{j \omega t} & (k=1,2), \\
q_{k}^{s M}(x, \theta, t)=M_{k}^{s} \sin \left(k_{n^{\prime}} x\right) \delta\left(\theta-\theta_{k}\right) e^{j \omega t} & (k=1,2),
\end{array}
$$

where $N_{k}^{s}$ and $Q_{k}^{s}$ are the amplitudes of forces per unit length, and $M_{k}^{s}$ is the amplitude of moment per unit length; $\delta\left(\theta-\theta_{k}\right)$ is the Dirac delta function.

Following the procedure described in part A, the shell's steady harmonic response excited by internal coupling forces represented by (22) can then be determined. Substituting (22) into (17) or (18) separately, modal participation factor $\lambda_{s m n}$ can be obtained. Combining it with (20), the respective dynamic displacements $u_{i}^{s}(i=1,2,3)$ of the shell are attained. The circumferential slope of the shell can be obtained from $u_{3}^{s}(x, \theta, t)$ by differentiation with respect to the circumferential coordinate $\theta$. With application of receptance definition, all expressions of receptances in matric $\left[\mathbf{Y}_{s c}\right]$ in (7) can be achieved.

2.2.3. Plate Receptances. The rectangular plate discussed here is supposed to be thin, uniform, and isotropic. Similar to the process of the shell, one can consider the receptances for a rectangular plate simply supported at all edges with forces and moments exerted at two joints: $\left(x, y_{1}\right)$ and $\left(x, y_{2}\right)$. According to the thin plate theory [25], under the influence of harmonic excitation and modal analysis, the displacement functions which satisfy all the boundary conditions are of the following form:

$$
\begin{aligned}
u_{1}^{p}(x, y, t) & =\sum_{m=1}^{\infty} \sum_{n=1}^{\infty} \lambda_{m n}^{p}(t) U_{1 m n}^{p}(x, y) \\
& =\sum_{m=1}^{\infty} \sum_{n=1}^{\infty} \lambda_{m n}^{p}(t) \cos \left(k_{n} x\right) \cos \left(k_{m} y\right), \\
u_{2}^{p}(x, y, t) & =\sum_{m=1}^{\infty} \sum_{n=1}^{\infty} \lambda_{m n}^{p}(t) U_{2 m n}^{p}(x, y) \\
& =\sum_{m=1}^{\infty} \sum_{n=1}^{\infty} \lambda_{m n}^{p}(t) \sin \left(k_{n} x\right) \cos \left(k_{m} y\right), \\
u_{3}^{p}(x, y, t) & =\sum_{m=1}^{\infty} \sum_{n=1}^{\infty} \lambda_{m n}^{p}(t) U_{2 m n}^{p}(x, y) \\
& =\sum_{m=1}^{\infty} \sum_{n=1}^{\infty} \lambda_{m n}^{p}(t) \sin \left(k_{n} x\right) \sin \left(k_{m} y\right),
\end{aligned}
$$

where $U_{1 m n}^{p}, U_{2 m n}^{p}$, and $U_{3 m n}^{p}$ are the rectangular plate's natural vibration modes in $x, y$, and $z$ directions, respectively; $k_{m}=m \pi / b, b$ is the width of the plate, and $k_{m}$ represent half wave numbers in the $y$ directions.
Substitute (23) into (16) and take consideration of isolation of the plate's transverse vibration and in-plane vibration, then

$$
N_{m n}^{p}= \begin{cases}\frac{b L}{4}, & \text { for transverse vibration, } \\ \frac{b L}{2}, & \text { for in-plane vibration. }\end{cases}
$$

For the plate-shell coupled system analyzed here, the joint line is at $\left(x, y_{1}\right)$ and $\left(x, y_{2}\right)$ in the plate's local coordinate. The line loads applied to the plate at each plateshell joint are forces $q_{k}^{p N}(x, y, t)(k=1,2)$ in $x$-axis direction and $q_{k}^{p Q}(x, y, t)(k=1,2)$ in $y$-axis direction and moments $q_{k}^{p M}(x, y, t)(k=1,2)$ around the $x$-axis. Similarly, all the line loads exerted on the plate at joints, as shown in Figure 3, can be expressed as follows:

$$
\begin{aligned}
q_{k}^{p N}(x, y, t) & =N_{k}^{p} \sin \left(k_{n^{\prime}} x\right) \delta\left(y-y_{k}\right) e^{j \omega t} \quad(k=1,2), \\
q_{k}^{p Q}(x, y, t) & =Q_{k}^{p} \sin \left(k_{n^{\prime}} x\right) \delta\left(y-y_{k}\right) e^{j \omega t} \quad(k=1,2), \\
q_{k}^{p M}(x, y, t) & =M_{k}^{p} \sin \left(k_{n^{\prime}} x\right) \delta\left(y-y_{k}\right) e^{j \omega t} \quad(k=1,2),
\end{aligned}
$$

where $N_{k}^{p}$ and $Q_{k}^{p}$ are the amplitudes of forces per unit length, $M_{k}^{p}$ is the amplitude of moment per unit length, $\delta\left(y-y_{k}\right)$ is the Dirac delta function, and $y_{k}(k=1,2)$ are the location of the joint line.

As for the external force $\left[\mathbf{F}_{p e}\right]$, it is assumed that only a point force $f_{p e}=F_{0} \delta\left(x-x_{0}\right) \delta\left(y-y_{0}\right) e^{j \omega t}$ is applied transversely to the plate. To get the related receptances in matric $\left[\mathbf{Y}_{p e}\right]$, it should be expressed in terms of a superposition of line forces of different order through fourier series expansion. After enough times of fourier series expansion, it can be expressed as follows:

$$
\begin{aligned}
f_{p e}(x, y, t)= & \frac{F_{0}}{L} \sum_{n^{\prime}=1}^{\infty}\left[\frac{2}{n^{\prime} \pi} \varepsilon\left(\frac{n^{\prime}}{2}\right)+\sin \left(k_{n^{\prime}} x_{e}\right)\right] \\
& \times \sin \left(k_{n^{\prime}} x\right) \delta\left(y-y_{e}\right) e^{j \omega t},
\end{aligned}
$$

where $\varepsilon\left(n^{\prime} / 2\right)=0$ if $n^{\prime}$ is odd, else $\varepsilon\left(n^{\prime} / 2\right)=1$.

Substitute (25) and (26) into (18) separately to get the related modal participation factor $\lambda_{s m n}$. Then expressions of respective responses for the plate due to these forces can be attained. According to the receptance definition, the plate's receptances matrix $\left[\mathbf{Y}_{p c}\right]$ in $(8)$ and $\left[\mathbf{Y}_{p e}\right]$ in (10) can be obtained.

\section{Numerical Studies}

For illustrative purposes, the plate/shell-coupled system is assumed to be defined by the following data set: density $7800 \mathrm{~kg} / \mathrm{m}^{3}$, Poisson's ratio 0.28 , and Young modulus $2.16 \times$ $10^{11} \mathrm{~N} / \mathrm{m}^{2}$. Thickness of the plate is $2 \times 10^{-3} \mathrm{~m}$, and the shell is $3 \times 10^{-3} \mathrm{~m}$. The shell's radius is $R=0.2 \mathrm{~m}$, length is $L=$ $1 \mathrm{~m}$, and angles of the two coupling edges in the cylindrical coordinate system are given as $\theta_{1}=\pi / 6$ while $\theta_{2}=5 \pi / 6$. 
3.1. Validation of the Theoretical Model. In order to validate the proposed model established with receptance method, comparisons between the present analysis and a wellestablished finite element analysis (FEA) using Ansys are performed. The FEA model was meshed with the shell 63 element, and its vibration response was obtained by harmonic analysis in Ansys. The external force is acted at $(L / 5, b / 2)$ on the plate, and $b$ is width of the plate. From the node deflections of an element, the spatial averaged squared surface acceleration was derived.

Figures 4 and 5 illustrate comparisons of both the plate's and the shell's average acceleration levels (referenced with $a_{\text {ref }}=10^{-6} \mathrm{~m} / \mathrm{s}^{2}$ ) obtained by Ansys and theory method separately. It is seen from Figures 4 and 5 that except for small discrepancies in the natural frequency values only small differences exist in the determination of the plate's and the shell's acceleration level between the theoretical predictions and those evaluated by the FEA approach, thus providing a measure of confidence in the validity of the computations. The reason for errors occurring in the natural frequencies lies in the fact that different plate-shell theories are used in the two calculations. That is, the influence of transverse shear deformation and rotational inertia of a plate-shell element is incorporated into the Ansys calculations, but they are ignored in the theoretical model. In previous studies $[13,14]$ related to free vibration of a cylindrical shell with an interior plate, it was found that errors also occurred in the prediction of natural frequencies for some modes between the theoretical method and the FEA approach. As shown in Figure 10, some errors are observed in the shell's acceleration level, because an approximation (i.e., (13)) is used to calculate the shell's acceleration.

3.2. Effect of the Plate's Location. In all calculations reported hereafter, the shell and the plate are both assumed to be made up of steel, whose material property has been described above. The structural parameters are as follows. For the shell, the radius is $R=0.2 \mathrm{~m}$, thickness is $h_{s}=0.003 \mathrm{~m}$, and length is $L=1 \mathrm{~m}$. The plate is excited by a unit harmonic force at $(L / 5, b / 2)$ in the plate's local coordinate.

Effect of the plate's location in the shell's cylindrical coordinate is discussed firstly. The plate's thickness is $h_{p}=$ $0.002 \mathrm{~m}$, and its location in the cylindrical co-ordinate system, as shown in Figure 2, is changed. Figures 6 and 7 present the average acceleration levels of the combined system with different locations of the interior plate, $\theta_{2}-\theta_{1}=$ $\pi / 4,2 \pi / 3$ and $\pi$.

It can be seen from Figures 6 and 7 that the first peak of both the shell and the plate's acceleration level curve shift to the lower frequency range when the plate is located closer to the center of the shell. This phenomenon implies the fundamental frequency of the combined shell decreases as the plate is located near the center of the plate. This can be attributed to the increase of the plate in the width as the first mode of the combined structure only involves the plate's bending mode [13].

In Figures 6 and 7, the general trend of the shell and the plate's acceleration level curves varies complicatedly with the changing of plate's location. For the plate, its acceleration

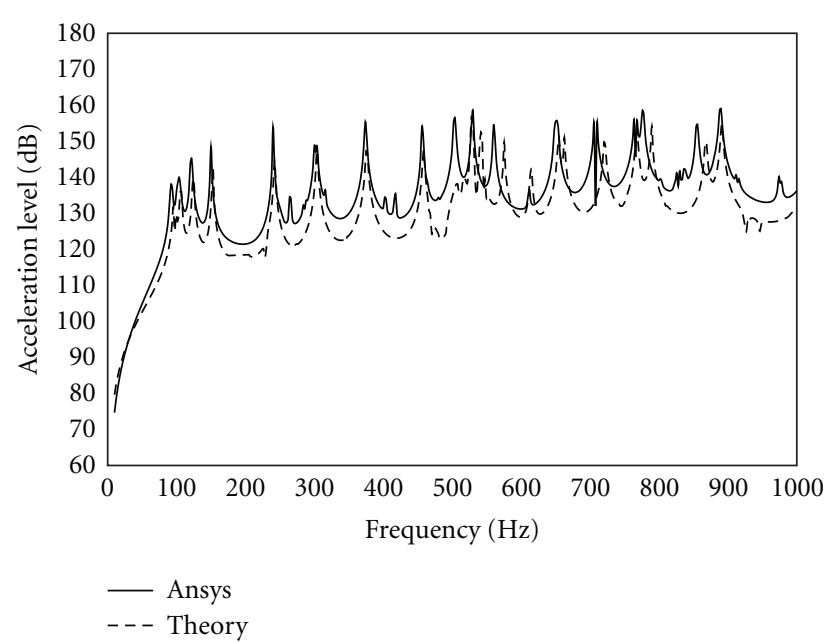

Figure 4: The plate's acceleration level for Ansys and theoretical results.

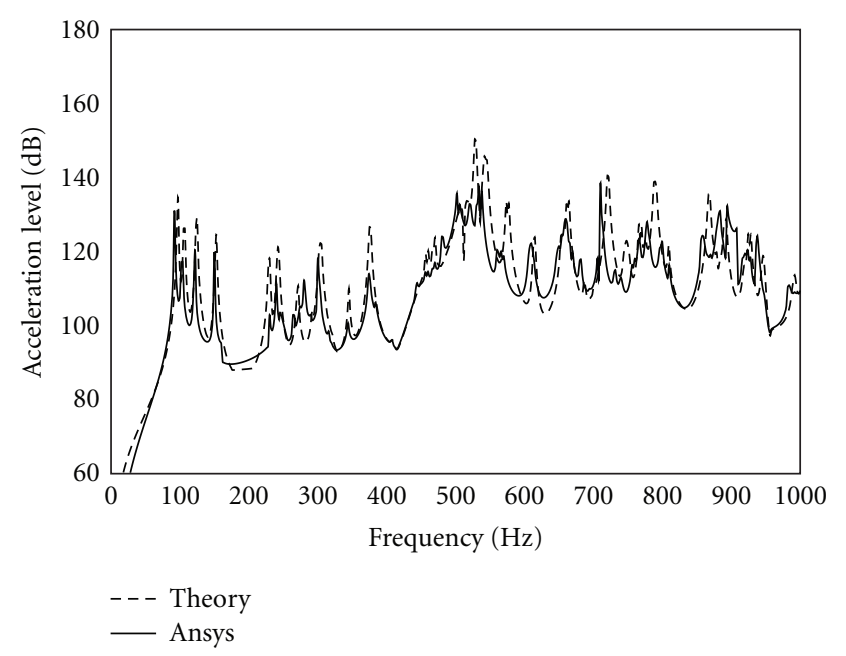

Figure 5: The shell's acceleration level for Ansys and theoretical results.

level mainly drops as the plate becomes wider, except in frequencies near $600 \mathrm{~Hz}$. The shell's acceleration level mainly, reversely, increases as the plate is located closer to the center of the shell, especially in the lower frequency range. This may be induced by the impedance features. According to the method described in Section 2.2, the driving-point impedance for the shell's and the plate's bending vibration can be obtained by application of a point force and expressed as follows:

$$
\begin{gathered}
\frac{1}{Z_{s}}=\frac{j \omega}{\rho_{s} h_{s}} \sum_{m=0}^{M} \sum_{n=1}^{N} \frac{\sin ^{2}\left(k_{n} x\right)}{N_{m n}\left[\omega_{s m n}^{2}\left(1+j \eta_{s}\right)-\omega^{2}\right]}, \\
\frac{1}{Z_{p}}=\frac{4 j \omega}{\rho_{p} h_{p} L b} \sum_{m=1}^{\infty} \sum_{n=1}^{\infty} \frac{\sin ^{2}\left(k_{n} x\right) \sin ^{2}\left(k_{m} y\right)}{\omega_{p m n}^{2}\left(1+j \eta_{p}\right)-\omega^{2}},
\end{gathered}
$$

where $Z_{s}$ is the driving-point impedance for the shell, and $Z_{p}$ is the driving-point impedance for the plate. 


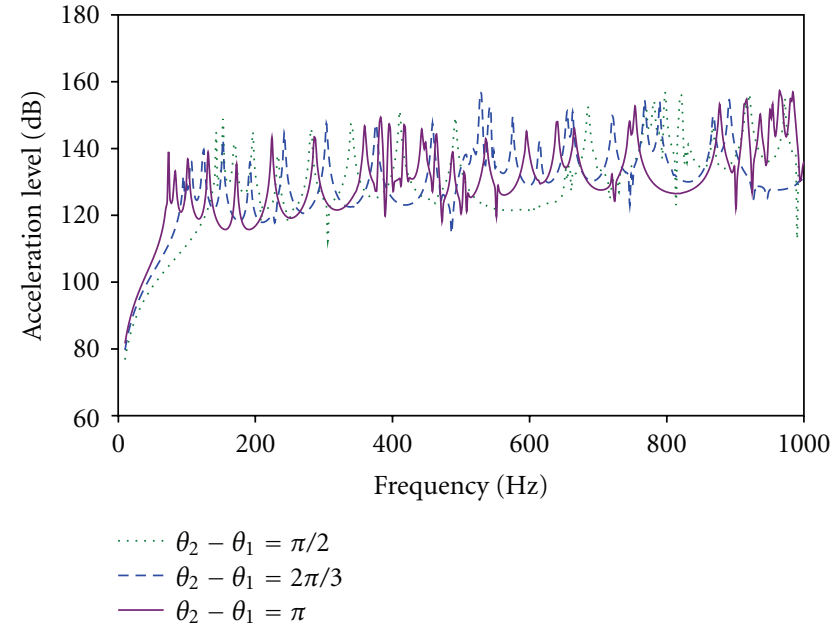

FIGURE 6: The plate's average acceleration level for different locations of the plate.

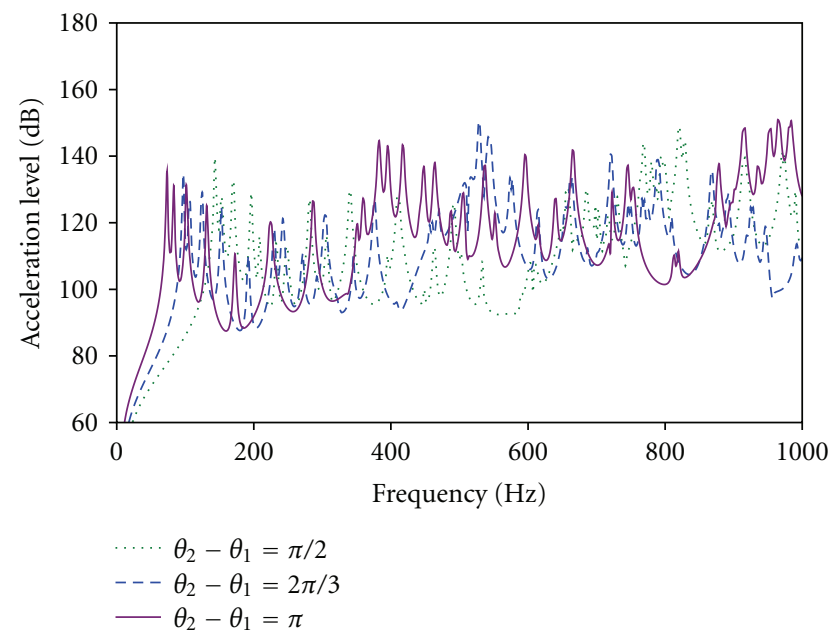

FIgURE 7: The shell's average acceleration level for different locations of the plate.

Equation (28) demonstrates that when the plate gets closer to the center of the shell, the width of the plate $b$ grows, and hence the plate's impedance increases. Comparison of the amplitudes of the shell and the plate's impedance is shown in Figure 8. It shows that $f_{0}=249 \mathrm{~Hz}$ is the first natural frequency of the shell. Impedance of the plate is smaller than that of the shell for the case here. As the plate's impedance increases when it becomes wider, impedances of the combined two substructures are more close to each other, which induces more vibration energy transmitting from the source plate to the receiver shell. Thus, increase of the shell's acceleration level is caused by impedance matching when the plate gets closer to the center of the shell.

3.3. Effect of the Plate's Thickness. It also can be seen from Figure 8 that, for the case discussed here, its largest impedance, which can be achieved when it is located at the

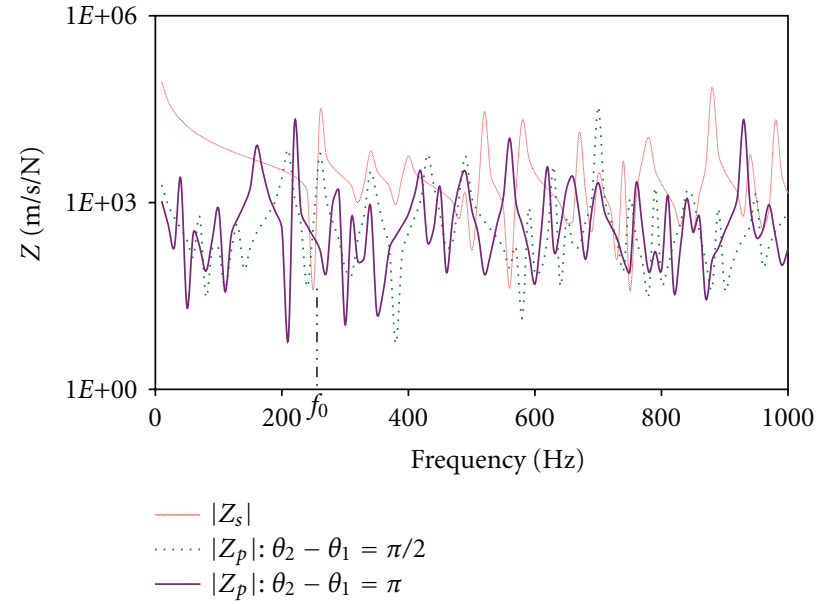

FIgure 8: Comparison of the shell and the plate's driving-point impedance for different locations of the plate.

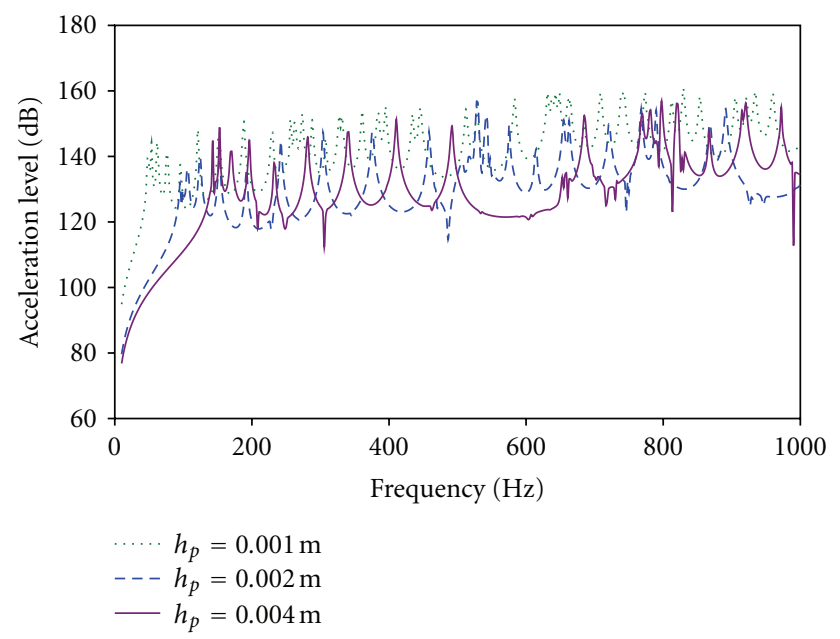

FIGURE 9: Average acceleration level of the plate for different thickness of the plate.

center of the shell, is still smaller than that of the shell. Another important factor influencing the plate's impedance is its thickness. In engineering applications, the shell's thickness is usually fixed. Thus, to reduce the vibration energy transmitted to the shell, it is useful to change the thickness of the plate under the condition that it satisfies the strength requirements. Figures 9 and 10 show how the plate and the shell's acceleration levels change with the variation of the plate's thickness, $h_{p}=0.001 \mathrm{~m}, 0.002 \mathrm{~m}$ and $0.004 \mathrm{~m}$.

From Figures 9 and 10, it can be seen that acceleration level of the plate drops greatly when its thickness increases, while the general trend of the shell after the first peak of each curve grows, except at some peak frequencies. The drop of the plate's acceleration level can be attributed to the increase of its impedance, which is easy to understand. To check whether the increase of the shell's acceleration level is still due to the approach of the plate and the shell's impedance, the driving-point impedances of these two 


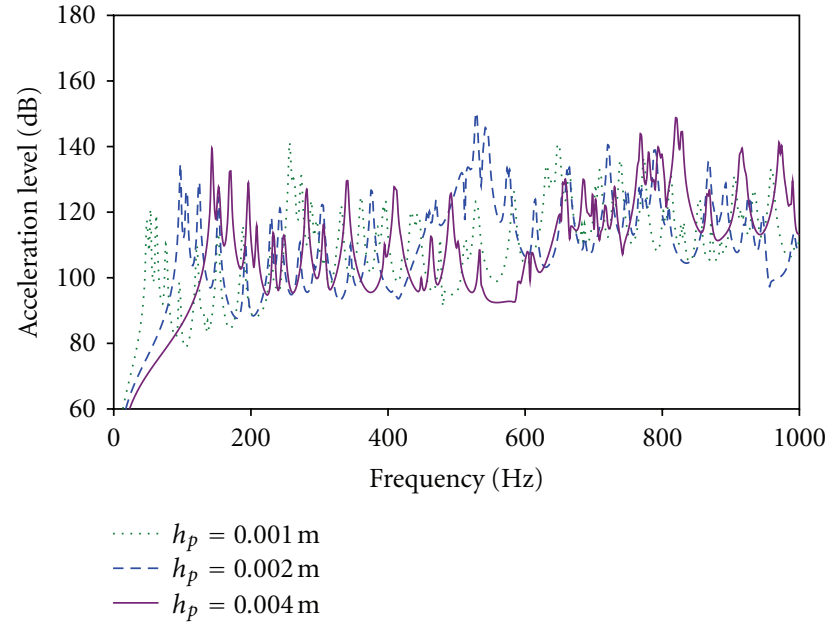

Figure 10: Average acceleration level of the shell for different thickness of the plate.

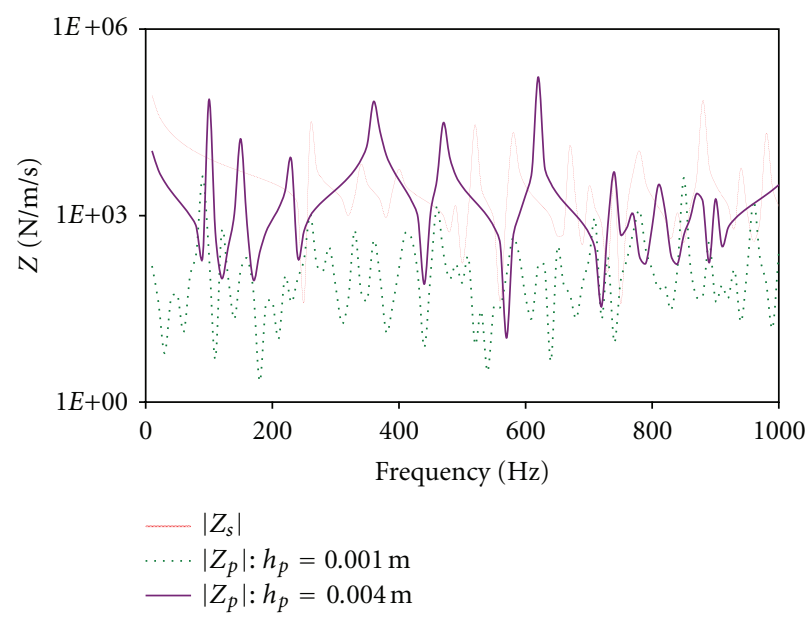

FIgURE 11: Comparison of the shell and the plate's driving-point impedance for different thickness of the plate.

substructures are compared again, as shown in Figure 11. It demonstrates that impedance of the plate increases as it becomes thicker. However, the plate's largest impedance here, when its thickness is $0.004 \mathrm{~m}$, is still not more than that of the shell. Therefore, the plate's impedance gets closer to that of the shell when its thickness increases. This validates the assumption that impedance matching would cause the increase of the shell's acceleration level is true.

According to the discussion above, it is reasonable to suppose that the acceleration level of the shell will drop at first when the thickness of the shell increases after it has passed that of the shell, and then it may tend to be stable when the impedance of the plate is far larger than the shell's. To confirm this hypothesis, acceleration levels of the plate and the shell with different thickness of the plate, $h_{p}=0.008 \mathrm{~m}, 0.016 \mathrm{~m}$, and $0.032 \mathrm{~m}$, are shown in Figures 12 and 13. It can be seen that both the plate and the shell's acceleration levels drop, especially at lower frequency range,

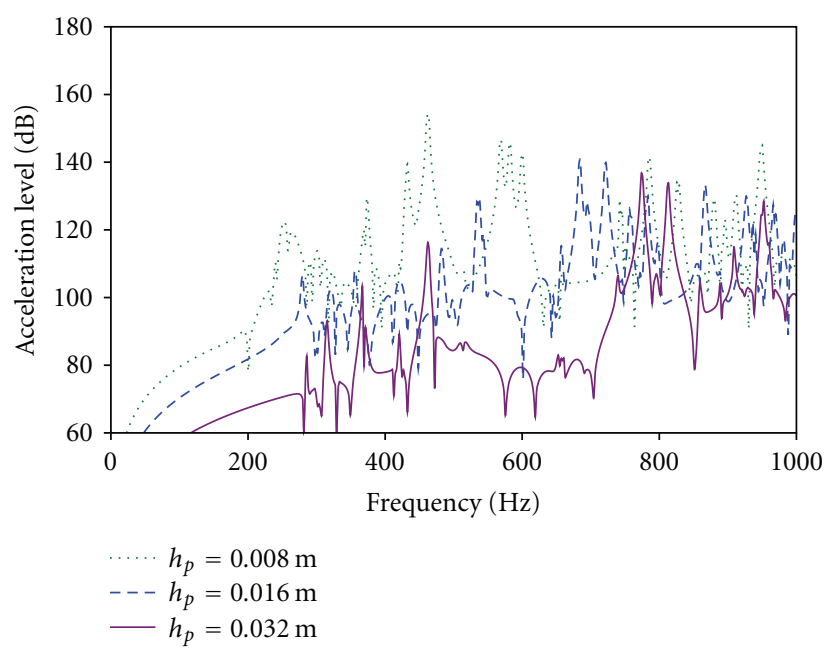

Figure 12: Comparison of the plate's average acceleration level.

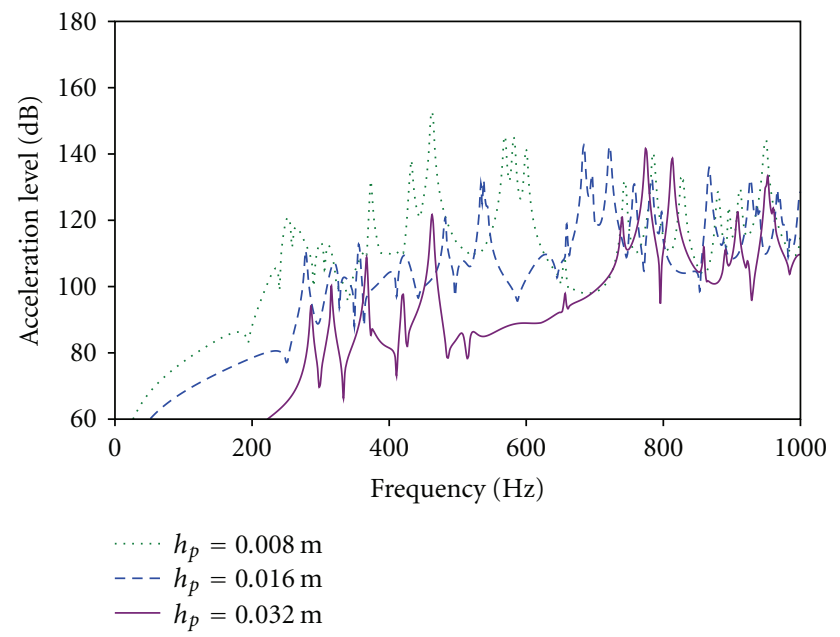

FIGURE 13: Comparison of the shell's average acceleration level.

as the thickness of the plate increases. However, at higher frequency range, the acceleration levels of the plate and the shell do not change very much as the thickness of the plate increases, after $800 \mathrm{~Hz}$ for this case. This might be caused by the shift of natural frequency for the coupled structure. The fundamental frequency of the coupled structure shifts to the higher frequency when the thickness of the plate increases. Thus, comparison of the acceleration levels is a little difficult because the peak of one curve may meet the decline of the other one at some frequencies. After all, the general trend of the shell's acceleration level decreases.

\section{Conclusions}

Vibration transmission characteristics of a cylindrical shell with a floor partition are analyzed with a receptance method. Utilizing the Love's shell and classical plate theory, receptance expressions of the plate and the shell are formulated. Then the frequency equation of the shell/plate combined system 
is obtained through considering geometrical compatibility conditions and force balance.

To validate the theoretical model, comparison of the theoretical results and values by FEA method shows that the theoretical model is effective to predict the vibration characteristics of the plate-shell-combined system. This also proves that the receptance method is practical for analysis of the forced vibration of the shell/plate-combined system.

As effects of structure parameters, including position and thickness of the plate, on vibration features of the system are of great importance for engineers in the design of suitable structures with low vibration and noise radiation characteristics, they are discussed in this paper. It shows that acceleration level of the plate decreases while that of the shell increases when the plate is located closer to the center of the shell due to the growth of the plate's impedance. Besides, when the impedance of the plate is smaller than that of the shell, and acceleration level of the plate drops significantly while that of the shell generally increases as the thickness of the plate increases; when the impedance of the plate is larger than that of the shell, acceleration levels of both the plate and the shell decrease as the thickness of the plate increases. Thus, it is important to avoid the impedance march of the each substructure to reduce the vibration energy transmitted from the source substructure to the receive substructure.

\section{Acknowledgments}

The authors would like to express their appreciation to Ren Jiean, Chen Yonghui, and Liu Rulin for assistance of the numerical experiments. This work is supported by a grant from Major Basic Research Development Program of China (973 Program) (Grant no. 613100).

\section{References}

[1] G. L. Giles, "Design-oriented analysis of aircraft fuselage structures using equivalent plate methodology," Journal of Aircraft, vol. 36, no. 1, pp. 21-28, 1999.

[2] Q. Zhou and P. F. Joseph, "A numerical method for the calculation of dynamic response and acoustic radiation from an underwater structure," Journal of Sound and Vibration, vol. 283, no. 3-5, pp. 853-873, 2005.

[3] C. Wang and J. C. S. Lai, "Prediction of natural frequencies of finite length circular cylindrical shells," Applied Acoustics, vol. 59, no. 4, pp. 385-400, 2000.

[4] L. Gan, X. Li, and Z. Zhang, "Free vibration analysis of ringstiffened cylindrical shells using wave propagation approach," Journal of Sound and Vibration, vol. 326, no. 3-5, pp. 633-646, 2009.

[5] F. Fahy and P. Gardonio, Sound and Structural Vibration, Elsevier Press, Amsterdam, The Netherlands, 2007.

[6] N. K. Mandal, R. A. Rahman, and M. S. Leong, "Experimental investigation of vibration power flow in thin technical orthotropic plates by the method of vibration intensity," Journal of Sound and Vibration, vol. 285, no. 3, pp. 669-695, 2005.

[7] M. R. Peterson and D. E. Boyd, "Free vibrations of circular cylinders with longitudinal, interior partitions," Journal of Sound and Vibration, vol. 60, no. 1, pp. 45-62, 1978.
[8] T. Irie, G. Yamada, and Y. Kobayashi, "Free vibration of noncircular cylindrical shells with longitudinal interior partitions," Journal of Sound and Vibration, vol. 96, no. 1, pp. 133142, 1984.

[9] R. S. Langley, "A dynamic stiffness technique for the vibration analysis of stiffened shell structures," Journal of Sound and Vibration, vol. 156, no. 3, pp. 521-540, 1992.

[10] J. Missaoui, L. Cheng, and M. J. Richard, "Free and forced vibration of a cylindrical shell with a floor partition," Journal of Sound and Vibration, vol. 190, no. 1, pp. 21-40, 1996.

[11] J. Missaoui and L. Cheng, "Vibroacoustic analysis of a finite cylindrical shell with internal floor partition," Journal of Sound and Vibration, vol. 226, no. 1, pp. 101-123, 1999.

[12] D. S. Li, L. Cheng, and C. M. Gosselin, "Analysis of structural acoustic coupling of a cylindrical shell with an internal floor partition," Journal of Sound and Vibration, vol. 250, no. 5, pp. 903-921, 2002.

[13] Y. S. Lee and M. H. Choi, "Free vibrations of circular cylindrical shells with an interior plate using the receptance method," Journal of Sound and Vibration, vol. 248, no. 3, pp. 477-497, 2001.

[14] Y. S. Lee, M. H. Choi, and J. H. Kim, "Free vibrations of laminated composite cylindrical shells with an interior rectangular plate," Journal of Sound and Vibration, vol. 265, no. 4, pp. 795817, 2003.

[15] Z. H. Wang, J. T. Xing, and W. G. Price, "A study of power flow in a coupled plate-cylindrical shell system," Journal of Sound and Vibration, vol. 271, no. 3-4, pp. 863-882, 2004.

[16] R. E. D. Bishop and D. C. Johnson, The Mechanics of Vibration, Cambridge University Press, Cambridge, UK, 1960.

[17] S. Azimi, J. F. Hamilton, and W. Soedel, "The receptance method applied to the free vibration of continuous rectangular plates," Journal of Sound and Vibration, vol. 93, no. 1, pp. 9-29, 1984.

[18] D. T. Huang and W. Soedel, "Natural frequencies and mode shapes of a circular plate welded to a circular cylindrical shell at arbitrary axial positions," Journal of Sound and Vibration, vol. 162, no. 3, pp. 403-427, 1993.

[19] D. T. Huang and W. Soedel, "On the Free vibrations of multiple plates welded to a cylindrical shell with special attention to mode pairs," Journal of Sound and Vibration, vol. 166, no. 2, pp. 315-339, 1993.

[20] D. T. Huang and W. Soedel, "Study of the forced vibration of shell-plate combinations using the receptance method," Journal of Sound and Vibration, vol. 166, no. 2, pp. 341-369, 1993.

[21] J. S. Tao, G. R. Liu, and K. Y. Lam, "Dynamic analysis of a rigid body mounting system with flexible foundation subject to fluid loading," Shock and Vibration, vol. 8, no. 1, pp. 33-47, 2001.

[22] M. Tursun and E. Ekinat, "Suppression of vibration using passive receptance method with constrained minimization," Shock and Vibration, vol. 15, no. 6, pp. 639-654, 2008.

[23] S. Werner, Vibration of Shells and Plates, Marcel Dekker, New York, NY, USA, 1993.

[24] L. Cremer, M. Heckl, and E. E. Ungar, Structure-Borne Sound, Springer, Berlin, Germany, 1988.

[25] J. T. Xlng and W. G. Price, "A power-flow analysis based on continuum dynamics," Proceedings of the Royal Society A, vol. 455, no. 1982, pp. 401-436, 1999. 

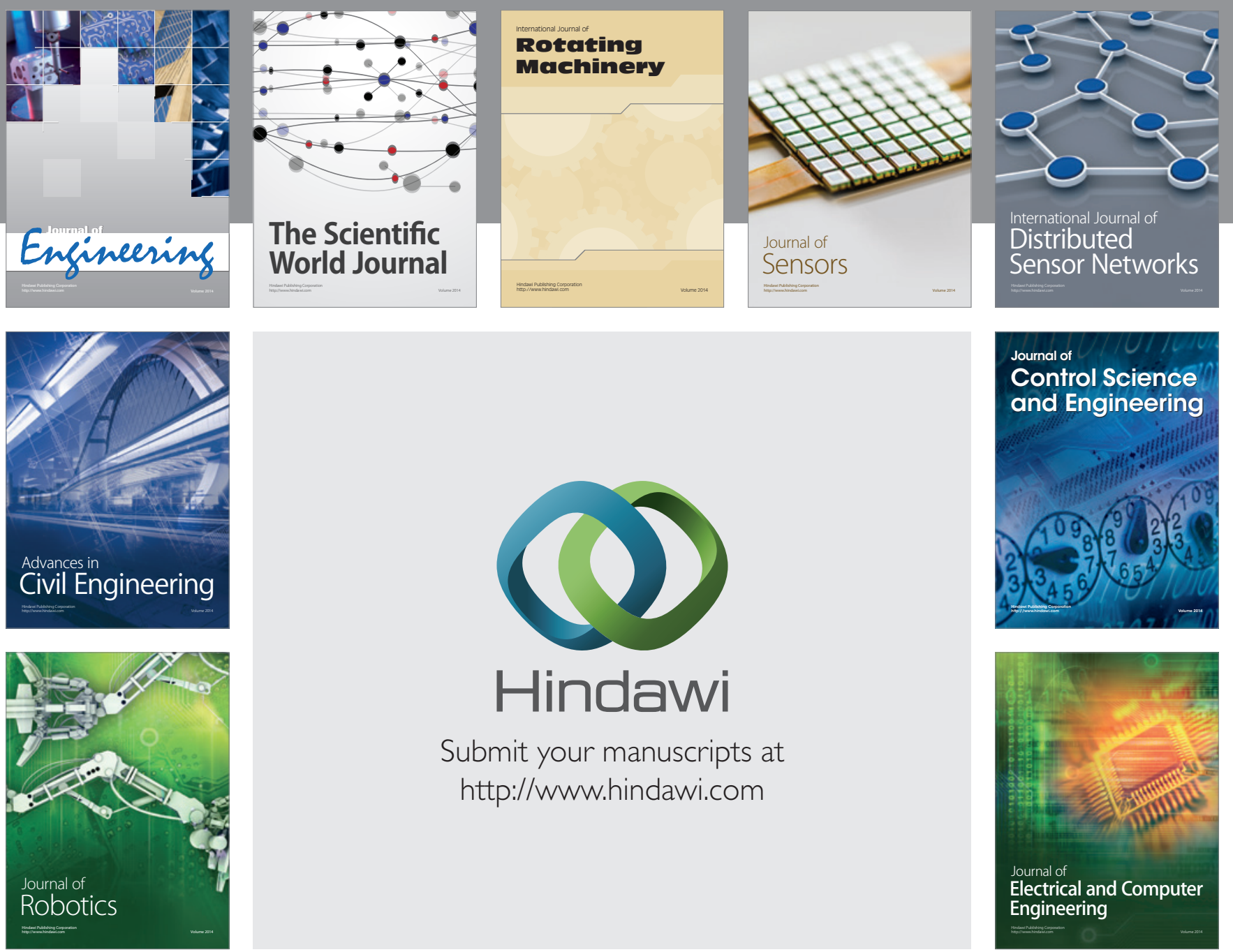

Submit your manuscripts at

http://www.hindawi.com
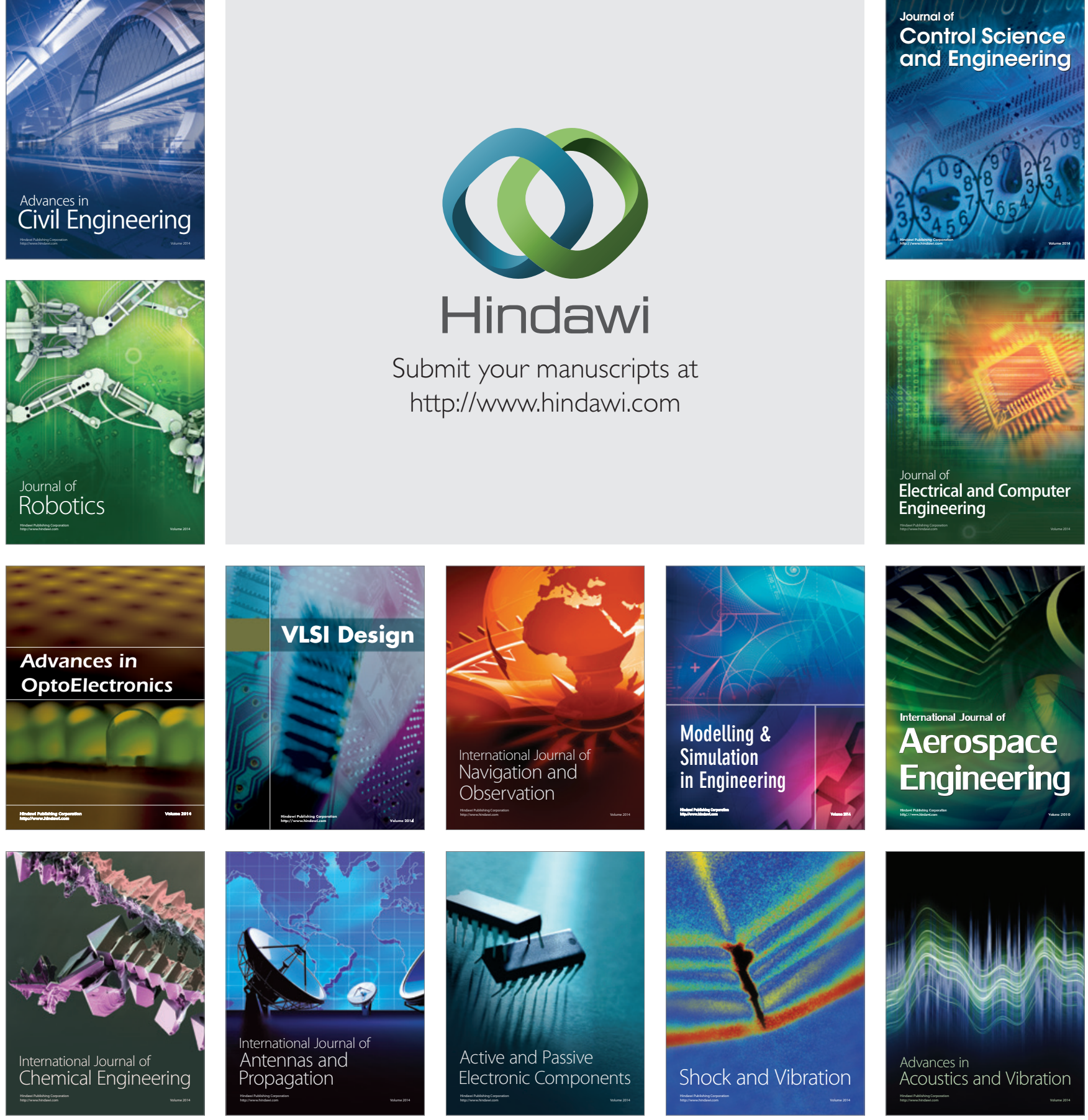